\title{
Coordinated expansion co-planning of integrated gas and power systems
}

\author{
Dongxiao WANG ${ }^{1}$, Jing QIU $^{1}$, Ke MENG ${ }^{2,3}$ (D), Xiaodan GAO ${ }^{1}$, \\ Zhaoyang DONG ${ }^{2,3}$
}

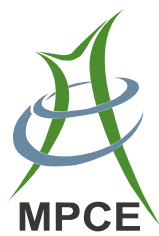

\begin{abstract}
As a significant clean energy source, natural gas plays an important role in modern energy context. The growing utilization of natural gas brings uncertainties into the power system, which requires an integrated way to plan natural gas and power systems. In this paper, the co-planning process is formulated as a mixed integer nonlinear programming problem to address emerging challenges, such as system reliability evaluation, market time line mismatch, market uncertainties, demand response effect, etc. An innovative expansion co-planning (ECP) framework is established in this paper to find the best augmentation plan which comes with the minimum cost. Specifically, to cope with uncertainties in market share,
\end{abstract}

CrossCheck date: 3 March 2017

Received: 3 January 2017/Accepted: 3 March 2017/Published online: 25 April 2017

(C) The Author(s) 2017. This article is an open access publication

$\triangle$ Ke MENG

kemeng@ieee.org

Dongxiao WANG

dong.wang@uon.edu.au

Jing QIU

qiujing0322@gmail.com

Xiaodan GAO

xiaodan.gao@uon.edu.au

Zhaoyang DONG

joe.dong@sydney.edu.au

1 Centre for Intelligent Electricity Networks, The University of Newcastle, Callaghan, NSW 2308, Australia

2 College of Electrical and Information Engineering, Changsha University of Science and Technology, Changsha 410114, China

3 School of Electrical and Information Engineering, The University of Sydney, Sydney, NSW 2006, Australia decision analysis is introduced. Meanwhile, the energy conversion efficiency between gas and electricity in the coupled load center is considered in the ECP constraints. Comprehensive case studies are applied to validate the performance of proposed approach.

Keywords Co-optimization, Expansion co-planning, Natural gas system

\section{Introduction}

Owing to the global concerns on climate change and worldwide carbon pricing policies, the consumption of natural gas has been ascending these years [1]. Compared with traditional fossil fuels, natural gas is believed to be more promising in a carbon-constrained world due to the distinct features brought by gas-fired power generation (GPG) units, such as lower emission intensity, faster response capability, etc. Plentiful research has been conducted towards predicting the proliferation of gas in power generation $[2,3]$. These researches indicate that GPG from the supply side tends to take a greater share in the electricity market and electricity users from the demand side are inclined to diversify the sources of energy usage due to the growing electricity price. The increasing penetration of GPG poses a new challenge on energy network congestion management and further requires an integrated planning framework to operate the power system securely and reliably.

In the literature, some researches have been done on the modelling of the integrated natural gas and power systems, in order to establish an optimal operation framework for the coupled system. Some researchers put emphasis on analysing the interdependency of energy networks for further evaluating system reliability $[4,5]$. The authors in 
[5] proposed an integrated model for analysing the interdependency of natural gas and power system security while incorporating the constraints of gas network into the optimal solution of security-constrained unit commitment. A few researches have been conducted on introducing energy flows to simulate operation planning [6-9]. As we can see, there are a number of constraints essential for the smooth operation of the coupled model, including pressure limits, nodal balance \& voltage, thermal limits, compressor working constraints, gas flow constraints, generation limits, and gas contracts and reserves [10-12]. These constraints are necessary for guaranteeing a secure and reliable coupled network. With regard to the integrated planning of power system generation and transmission, some works are found in [13-15]. However, the fuel availability and fuel network operating constraints are neglected in these studies. The authors in [16] presented an expansion planning model for electricity and networks, which was formulated as a long-term, multi-area and multi-stage problem. Nevertheless, the authors merely considered the system value chain and the optimal dispatch of existing and new facilities, but did not define clear criteria for system performances. The integrated planning under market conditions are conducted in [17, 18]. In [17], the authors considered the expansion co-planning with uncertainties (e.g. uncertainties of energy load, load percentages, and market price) in a coupled energy market. In [18], the authors studied the expansion co-planning in terms of shale gas and power systems in a combined energy market, aiming to minimize the investment costs and improve the overall system reliability.

With the application of smart grids, demand response (DR) programs have attracted significant attentions in terms of enhancing system efficiency [19, 20]. According to [21], DR programs are categorized into incentive-based DR (IBDR) (e.g. emergence demand response program, direct load control, interruptible/curtailable service, ancillary service market, capacity market, and demand bidding) and price-based DR (e.g., time-of-use, real time pricing and critical peak pricing). On the other hand, when DR reaches a critical market level, the uncertainty of consumer behaviour makes transmission expansion planning more challenging. Therefore, the influence of DR on system security and adequacy should be comprehensively investigated. The authors in [20] considered the impact of DR resources on transmission expansion planning, especially in terms of system security. However, the DR impacts on gas and electricity expansion co-planning have not been addressed in detail in previous work.

By reviewing the literature, we propose an expansion co-planning (ECP) framework in a combined electricity market to address the above challenges. In this ECP model, the responsibility of planning the coupled natural gas and power system is undertaken by an independent system operator (ISO), which takes a similar role to Australian Energy Market Operators (AEMO) [22]. AEMO manages the Australia national electricity and gas markets through regulating the third party, which executes the function of maintaining and augmenting the system infrastructure. Focusing on providing planning suggestions with regard to network capability and constraints, AEMO leaves the investment decision to involved third party asset owners. From the point of ISO, the ECP model is a co-optimization problem, reflecting the non-linear and non-convex characteristics of integrated systems. The model is formulated for the sake of minimizing the total cost of gas and power sectors, in the meanwhile, meeting a variety of technical constraints. It is worth noting that in this research, the energy conversion efficiency between gas and electricity is considered in the ECP constraints as well. The innovative contributions of this paper are summarized as threefold: 1) Decision analysis is adopted to deal with uncertainties of the combined market in the planning process; 2) By using the method of selecting the maximum benefit ratio, augmentation options regarding capital investments, expenditure reductions and market benefits are compared; 3) DR impacts on gas and electricity expansion co-planning are detailed addressed in this research.

This paper is organized as follows. In Section 2, the modelling of natural gas system is presented. Section 3 gives the expansion co-planning framework, subject to a variety of technical constraints. In Section 4, the simulation studies are performed on two coupled energy systems. Finally, Section 5 concludes this paper.

\section{Natural gas network model}

\subsection{Linepack equations}

Linepack (LP) refers to a procedure that allows the pressurized gas into a pipeline throughout the gas network [23]. It is regarded as a key factor that affects the capability of supplying gas to demand nodes (i.e. fluctuations in demand are allowed in a highly packed pipe). The gas equation state is:

$\frac{\rho_{0}}{\psi_{0} \Gamma_{0}}=\frac{\rho}{\psi \Gamma}=Z R_{\text {gas }}=$ Constant

where $\rho$ is gas pressure $(\mathrm{kPa}) ; \psi$ is gas density $\left(\mathrm{kg} / \mathrm{m}^{3}\right) ; \Gamma$ is temperature $(\mathrm{K}) ; \rho_{0}, \psi_{0}, \Gamma_{0}$ are gas pressure, gas density, and temperature under normal conditions, respectively; $Z$ is gas constant compressibility factor; $R_{\text {gas }}$ is gas constant $(\mathrm{J} /$ $(\mathrm{kg} \mathrm{K})$ ).

According to Boyle's law, we have: 
$\rho_{i j}^{\text {aver }} H_{i j}^{\text {aver }}=\rho_{0} H_{0}$

where $\rho_{i j}^{a v e r}$ is the average pressure of a pipe at steady state between node $i$ and node $j ; H_{i j}^{a v e r}$ is the pipe volume between nodes $i-j$, which is equal to the gas volume; $H_{0}$ is the pipe volume under normal conditions.

Based on (1) and (2), we get the initial LP in a steady state under normal conditions:

$\Pi_{i j}^{\text {initial }}=\frac{\rho_{i j}^{\text {aver }} H_{i j}^{\text {aver }}}{\psi_{0} \Gamma_{0} Z R_{\text {gas }}}$

The dynamic LP is expressed as:

$\Pi_{i j}(t)=\Pi_{i j}^{\text {initial }}+\int_{0}^{t}\left[P_{s u p, i}^{\text {gas }}(t)-P_{\text {con }, i}^{\text {gas }}(t)\right] \mathrm{d} t$

where $P_{s u p}^{g a s}$ is supplied gas in a pipe; $P_{c o n}^{g a s}$ is consumed gas in a pipe.

\subsection{Gas flow}

Bernoulli fluid equation is used here for describing the gas flow along a horizon pipe in a steady state, as [9]:

$S_{i j}^{g a s}=\operatorname{sgn}_{i j} K \frac{\Gamma_{0}}{\rho_{0}} \sqrt{\operatorname{sgn}_{i j} \frac{\left(\rho_{i}^{2}-\rho_{j}^{2}\right) D_{i j}^{5}}{F_{i j} G^{\prime} L_{i j} \Gamma Z}}$

$K=\sqrt{\frac{\pi^{2} R_{\text {air }}}{64}}=3.2387$

$\operatorname{sgn}_{i j}=\left\{\begin{array}{cc}+1 & \text { if } \rho_{i}-\rho_{j}>0 \\ -1 & \text { if } \rho_{i}-\rho_{j}<0\end{array}\right.$

where $S_{i j}^{\text {gas }}$ denotes gas flow; $\operatorname{sgn}_{i j}$ are directions, which are decided by nodal pressure differences; $K$ is a constant determined by air constant $R_{\text {air }} ; \rho_{i}$ and $\rho_{j}$ are gas pressure at node $i$ and node $j ; D_{i j}$ is internal diameter; $F_{i j}$ is the friction factor decided by $D_{i j}$ [6]; $G^{\prime}$ is the gravity ratio defined as $R_{\text {air }} / R_{\text {gas }} ; L_{i j}$ is the pipeline length between node $i$ and node $j$.

\subsection{Gas compressor}

The complexity of gas system modelling is specifically caused by the non-linear and non-convex nature of compressors [24]. The empirical equation for the compressor power is expressed as:

$P_{C, c}=\frac{S_{C, c} \phi}{\eta_{c}(\phi-1)}\left[\left(\frac{\rho_{c}^{\text {out }}}{\rho_{c}^{\text {in }}}\right)^{\frac{\phi-1}{\phi}}-1\right]$

where $P_{C, c}$ is the power of compressor $c\left(10^{5} \mathrm{~W}\right) ; S_{C, c}$ is the gas volumetric flow rate at compressor $c ; \varphi$ is the polytropic exponent of the empirical equation; $\eta_{c}$ is the comprehensive efficiency ratio for compressor $c ; \rho_{c}^{\text {out }}$ and $\rho_{c}^{i n}$ are the compressor outlet and inlet pressures $(\mathrm{Pa})$, respectively.

\subsection{Gas and electricity market interaction}

There are two parts in the natural gas market, one is the financial market and the other is the physical market. The financial market involves trading of prospective contracts, while the physical market is based on the gas flow volume for the practically delivered gas at a particular delivery point. In Australia natural gas market structure, the gas trading action is completed based on per day cycle. Normally, the dispatch for the natural gas is decided by the cost order, which means the cheapest gas is served first until the load is met [22]. Four supplies reschedule in the next day, at 10.00 AM, 2.00 PM, 6.00 PM, and 10.00 PM respectively. However, determined by the Australia National Electricity Market (ANEM), the dispatch interval for electricity is five minutes.

The mismatch in timeline brings uncertainties to the available fuel amount in gas-powered generation units and further affects the reliable operation of the coupled gas and electricity network. One solution to offset the fluctuating gas demand is by gas storage, e.g. linepack. As the most promising gas storage method, linepack is replenished when injections are larger than demands, and linepack is consumed when injections are lower than demands.

\section{Formulation of expansion co-planning model}

An expansion co-planning framework is proposed in this paper considering the interdependency of natural gas systems and power systems, shown in Fig. 1. A variety of planning assumptions are assessed in this framework, including the developments of power generation, gas supply, together with the growth in load. In addition, the criteria for system planning and systematic performance requests are established here to identify the optimal augmentation option for reliably and safely supplying energy over the entire outlook period. The characteristics of coupled natural gas and electricity systems are demonstrated in two forms: 1) coupled load centers, where electricity and gas can be mutually transferred under some circumstances; 2) coupled generation centers, where GPG performs the role of gas loads and simultaneously the role of power suppliers. In this paper, four elements are taken into account in the planning process: GPG units, compressor stations, gas pipelines and electricity transmission lines.

The method of separated gas and power system expansion planning (EP) is briefly clarified here for better understanding. As we can see from Fig. 1, there are three 


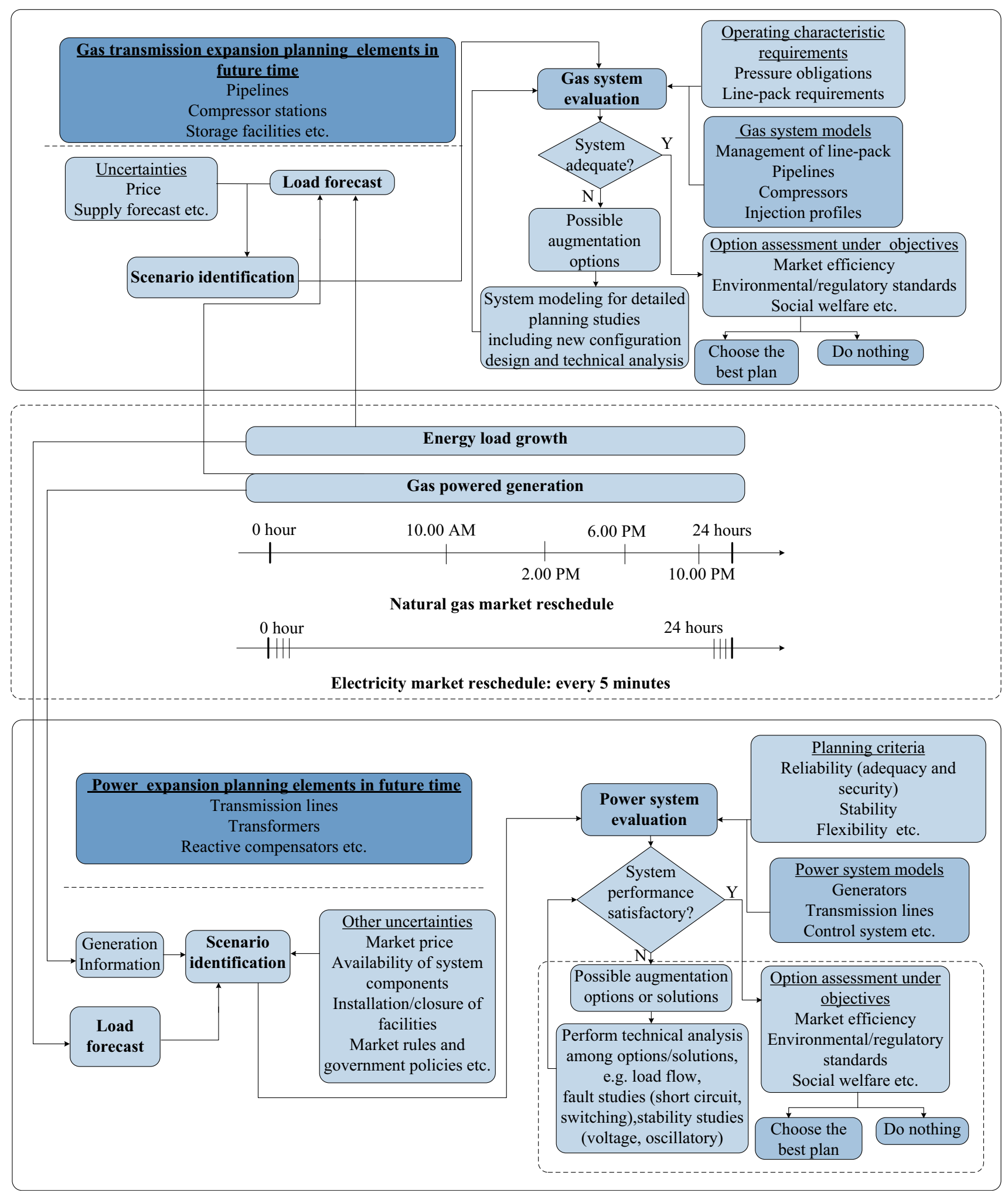

Fig. 1 Proposed expansion co-planning framework for coupled gas and electricity network

parts in total: the upper part denotes gas transmission expansion planning elements in future time; the middle part denotes coupled elements in the ECP framework and the market interaction; the lower part denotes power transmission expansion planning elements in future time. In the situation of EP framework, the middle part is eliminated, 
and only the upper and lower parts are kept. The gas transmission expansion planning aims to determine the size and location of pipelines, compressor stations, and storage facilities, while the power expansion planning tries to find the idea size and location of transmission lines, transformers, etc. These processes are conducted separately without considering coupled load centers and coupled generation centers. For detailed EP method, please refer to the upper/lower part in Fig. 1.

\subsection{Detailed expansion co-planning model}

The formulated objective is to minimize the total cost of the gas and power sectors, including investment capital $\operatorname{cost} \boldsymbol{C}_{\text {cap }}$, operation cost $C_{\text {oper }}$, and DR cost $C_{D R}$. For simplicity, our planning is a single stage and all financial indices are estimated on the same time scale (i.e. an annual basis). The model is expressed as:

$\min \boldsymbol{\beta}^{\mathrm{T}} \boldsymbol{C}_{c a p}+C_{\text {oper }}+C_{D R}$

where $\boldsymbol{\beta}$ is a vector representing the binary decision variables (' 1 ' is executing augmentation; ' 0 ' is doing nothing); $\boldsymbol{C}_{c a p}$ is a vector denoting the corresponding annualized capital investment costs of $\boldsymbol{\beta}$. It is assumed that the costs of planning components depending either on lengths, like gas pipelines and power lines, or capacities, such as GPG units and compressor stations. The capital costs are further expressed as:

$\boldsymbol{\beta}^{\mathrm{T}} \boldsymbol{C}_{\text {cap }}=\sum\left(\beta_{l n}^{\text {elec }} C_{l n}^{\text {elec }}+\beta_{i}^{G P G} C_{i}^{G P G}+\beta_{i}^{G C} C_{i}^{G C}+\beta_{i j}^{g a s} C_{i j}^{\text {gas }}\right)$

where $\beta_{l n}^{\text {elec }}, \beta_{i}^{G P G}, \beta_{i}^{G C}$ and $\beta_{i j}^{g a s}$ are decision variables for the power line, the GPG unit, the compressor, and the gas pipeline; $C_{l n}^{\text {elec }}, C_{i}^{G P G}, C_{i}^{G C}$ and $C_{i j}^{\text {gas }}$ are capital costs of additional power line $l-n$, additional GPG unit at node $i$, additional compressor at $i$ and additional gas pipeline $i-j$.

The detailed calculations of the remaining terms in (9) are given in (11) and (12):

$$
\begin{aligned}
C_{\text {oper }}= & \sum_{k \in N_{G}} \sum_{t=1}^{8760}\left(b_{1 k} P_{G, k t}^{2}+b_{2 k} P_{G, k t}+b_{3 k}\right) \\
& +\sum_{g \in N_{P}} \sum_{t=1}^{8760}\left(\Psi_{g} P_{P, g t}^{g a s}\right) \\
& +\sum_{c \in N_{C}} \sum_{t=1}^{8760}\left(a_{1 c} P_{C, c t}^{2}+a_{2 c} P_{C, c t}+a_{3 c}\right)
\end{aligned}
$$

where $N_{G}, N_{P}, N_{D}$ and $N_{C}$ are sets for nodes with power generators, gas producers, all demand nodes and gas compressors, respectively; $b_{1 k}, b_{2 k}, b_{3 k}$ are the first, second, third order cost coefficients of power generator $k$;
$P_{G, k t}$ is generated active power at time $t$ at generator $k ; \Psi_{g}$ is the cost coefficient of gas production; $P_{P, g t}^{g a s}$ is the gas production amount at time $t$ at producer $g ; P_{C, c t}$ is the compressor $c$ power at time $t ; a_{1 c}, a_{2 c}, a_{3 c}$ are coefficients of compressor $c$ operation cost.

$C_{D R}=\sum_{d \in N_{D}} \sum_{t=1}^{8760}\left(\alpha_{1 d} P_{D R, d t}^{2}+\alpha_{2 d} P_{D R, d t}+\alpha_{3 d}\right)$

where $\alpha_{1 d}, \alpha_{2 d}, \alpha_{3 d}$ are cost coefficients of DR in the power sector; $P_{D R, d t}$ denotes DR active power at time $t$ bus $d$.

Be noted that this paper mainly focuses on incentivebased DR, i.e. controllable/interruptible load (IL). System operators have the contractual authority to adjust customer power load directly when necessary. Customers receive financial compensations if they reduce or increase electricity consumption voluntarily when requested. System operators sign a contract with controllable/interruptible consumers, in which the upper limit and cost of load curtailment are specified. A quadratic polynomial function is one of the commonly used functions to model the cost of IL in the literature, such as [25] and [26]. For this reason, we adopt (12) to denote the DR cost in this research.

\subsection{Constraints}

Objective (9) is subject to the following constraints. In order to avoid the repeated information, the subscript $t$ is removed in the constraints.

1) Power balance constraint

$P_{G, k}-\left(P_{D, l}^{\text {elec }}-P_{D R, d}\right)=\sum_{n=1}^{N}\left|Y_{l n} V_{l} V_{n}\right| \cos \left(\theta_{l n}+\delta_{n} \delta_{l}\right)$

$Q_{G, k}-Q_{D, l}^{\text {elec }}=\sum_{n=1}^{N}\left|Y_{l n} V_{l} V_{n}\right| \sin \left(\theta_{l n}+\delta_{n}-\delta_{l}\right)$

where $P_{G, k}, Q_{G, k}$ are generator $k$ active and reactive power outputs; $P_{D, l}^{\text {elec }}, Q_{D, l}^{\text {elec }}$ denote the predicted active and reactive electricity demand; $V_{l}, V_{n}$ are bus voltages at node $l$ and node $n ; \delta_{l}, \delta_{n}$ are voltages angles; $Y_{l n}$ is the element in power system admittance matrix, and $\theta_{l n}$ is the angle of $Y_{l n}$. 2) Electricity branch flow constraint

$\underline{S}_{l n}^{\text {elec }} \leq S_{l n}^{\text {elec }} \leq \bar{S}_{l n}^{\text {elec }} \quad \forall l, n \in N_{\text {power }}$

where $S_{l n}^{\text {elec }}$ is power flow between branch $l-n ; \underline{S}_{l n}^{\text {elec }}$ and $\bar{S}_{l n}^{\text {elec }}$ represent lower and upper bounds of power flow; $N_{\text {power }}$ is the number of electricity bus node.

3) Nodal voltage constraint

$\underline{V}_{l} \leq V_{l} \leq \bar{V}_{l} \quad \forall l \in N_{\text {power }}$ 
where $V_{l}$ is voltage magnitude at node $l ; \underline{V}_{l}$ and $\bar{V}_{l}$ are the lower and upper limits for voltage

4) Power generator and DR constraint

$$
\begin{aligned}
& \left\{\begin{array}{l}
\underline{P}_{G, k} \leq P_{G, k} \leq \bar{P}_{G, k} \\
\underline{Q}_{G, k} \leq Q_{G, k} \leq \bar{Q}_{G, k}
\end{array} \quad \forall k \in N_{G}\right. \\
& \underline{P}_{G, k} \leq P_{G, k} \leq P_{D, i}^{g a s} \hbar \quad \forall i \in N_{G P G} \\
& \left|\frac{P_{D R, d}}{P_{D, l}}\right| \leq \zeta
\end{aligned}
$$

where $\underline{P}_{G, k}, \bar{P}_{G, k}$ represent lower and upper bounds for active power output of generator $k ; \underline{Q}_{G, k}, \bar{Q}_{G, k}$ represent lower and upper bounds for generator $k$ reactive power output; $P_{D, i}^{\text {gas }}$ is gas load of GPGs at node $i$; $\hbar$ is the gas heat rate; $P_{D, l}$ is power demand at bus $l ; \zeta$ is DR ratio. Equation (18) denotes that the outputs of GPGs are constrained by the availability of gas in gas networks. Equation (19) states DR is bounded by the maximum DR ratio $\zeta$.

5) Electricity network topology constrains

$$
\begin{aligned}
& Y_{l l}=y_{l l}^{0}+\sum\left(y_{l n}^{0}+\beta_{l n}^{\text {elec }} \gamma_{l n}\right) \quad l \neq n \\
& Y_{l n}=-\left(y_{l n}^{0}+\beta_{l n}^{\text {elec }} \gamma_{l n}\right) \quad l \neq n
\end{aligned}
$$

where $y_{l l}^{0}, y_{l n}^{0}$ represent the old admittance matrix elements; $Y_{l l}, Y_{l n}$ are the new admittance matrix elements after expansion planning; $\gamma_{l n}$ is the new circuit admittance.

6) Nodal gas flow constrains

$$
\begin{aligned}
& P_{P, i}^{\text {gas }}+P_{s u p, i}^{\text {gas }}+\sum S_{i j}^{\text {gas }}=\sum S_{j i}^{\text {gas }}+P_{c o n, i}^{\text {gas }}+\hbar P_{C, c} \\
& \quad \forall i, j \in N_{g a s} \\
& P_{P, g}^{\text {gas }} \leq P_{P, g}^{\text {gas }} \leq \bar{P}_{P, g}^{\text {gas }} \quad \forall g \in N_{P} \\
& \mu^{\mathrm{min}} \leq \frac{P_{D, l}^{\text {elec }}}{P_{D, l}^{\text {elec }}+\lambda^{g e} P_{c o n, i}^{\text {gas }}} \leq \mu^{\max }
\end{aligned}
$$

where $P_{\text {sup }, i}^{\text {gas }}, P_{\text {con }, i}^{\text {gas }}$ are natural gas supplied and consumed amount at node $i ; \sum S_{i j}^{\text {gas }}$ is gas flow between brand branch $i-j$. Equation (22) signifies the gas nodal balance at $i$. Equation (23) defines natural gas production constraints, where $P_{P, i}^{g a s}, \underline{P}_{P, i}^{g a s}, \bar{P}_{P, i}^{g a s}$ represent gas production at generator $i$, the lower generation limit and the upper generation limit, respectively. Equation (24) denotes the constraints of energy conversion ratio between gas demand and electricity demand, where $\lambda^{g e}$ represent the conversion ratio $(\mathrm{MWh} /$ $\left.\mathrm{m}^{3}\right), \mu^{\min }$ and $\mu^{\max }$ are the lower and upper bounds.

It should be noted that in the research, we only considered the coupling mechanism of Gas to Power (G2P), without taking into account the coupling of Power to Gas (P2G). Equation (18) and Equation (24) indicate the constraint of gas-fired power generations outputs and the constraint of energy conversion ratio between gas demand and electricity demand, respectively. By considering the constraints in generation side (i.e. (18)) and demand side (i.e. (24)), we decree that the coupling mechanism of G2P is guaranteed in this paper. The coupling mechanism of P2G will be investigated in our future work. In such a situation, the bi-directional energy conversion would be included in the model. Existing research, such as [27], has demonstrated the benefits of $\mathrm{P} 2 \mathrm{G}$ on transmission loss reduction and increasing renewable integration, making it necessary to consider both G2P and P2G in natural gas and power system co-planning.

7) Gas network technical constrains

$\underline{S}_{i j}^{\text {gas }} \leq S_{i j}^{\text {gas }} \leq \bar{S}_{i j}^{\text {gas }} \quad \forall i, j \in N_{\text {gas }}$

$\underline{\rho}_{i} \leq \rho_{i} \leq \bar{\rho}_{i} \quad \forall i \in N_{\text {gas }}$

$1<\frac{\rho_{c}^{\text {out }}}{\rho_{c}^{\text {in }}} \leq \bar{\xi}_{c} \quad \forall c \in N_{C}$

$\rho_{c}^{i n} \geq \underline{\rho}_{c}^{i n} \quad \forall c \in N_{C}$

$\rho_{c}^{\text {out }} \leq \bar{\rho}_{c}^{\text {out }} \quad \forall c \in N_{C}$

$S_{C, c} \leq \bar{S}_{C, c} \quad \forall c \in N_{C}$

$P_{C, c} \leq \bar{P}_{C, c} \quad \forall c \in N_{C}$

Other gas network constraints (1)-(8)

where $S_{i j}^{g a s}, \underline{S}_{i j}^{g a s}, \bar{S}_{i j}^{g a s}$ are gas flow capacity, the lower bound of gas flow capacity and the upper bound of gas flow capacity, respectively. Equation (26) constraints nodal pressure, where $\rho_{i}, \underline{\rho}_{i}, \bar{\rho}_{i}$ are nodal pressure, the lower limit of nodal pressure and the upper limit of nodal pressure. Equation (25) limits gas flow capacity. Equations (27)-(29) constraint compressors pressure, where $\rho_{c}^{i n}, \underline{\rho}_{c}^{i n}$ are the inlet pressure of a compressor and the lower limit of inlet pressure; $\rho_{c}^{\text {out }}, \bar{\rho}_{c}^{\text {out }}$ are the outlet pressure of a compressor and the upper limit of outlet pressure; $\bar{\xi}_{c}$ is the upper compressor pressure ratio limit. Equations (30) and (31) express the constraints of compressor flow and power, where $S_{C, c}, \bar{S}_{C, c}$ are gas flow rate at compressor $c$ and the upper limit of gas flow; $P_{C, c}, \bar{P}_{C, c}$ are power for compressor $i$ and the upper limit of power.

\section{8) Reliability constrains}

$E U E \leq \overline{E U E}$

Equation (32) states a reliability criterion, i.e., expected unserved energy (EUE). It is indispensable to assess the system security and reliability in expansion co-planning. As a common network security criterion, the $N-1$ norm has already been extensively used in the power system, i.e., the system is capable of functioning normally given a 
failure of one individual part. However, the $N-1$ criterion is not applicable to the gas system, as no such criterion has been found in gas systems. Given the necessity of adequacy evaluation for the coupled system, therefore, the adoption of EUE is proposed as a criterion to evaluate the reliability for both gas and electricity transmission networks. EUE refers to the predicted quantity of energy which is not provided because of the insufficient capacities for both generation and transmission. Note that we only consider the energy demand from the compromised end-users, which implies the curtailed gas demand of GPG under the gas supply constraints is not included.

\subsection{Uncertainties in combined market}

Numerous uncertainties exist in the deregulated combined electricity and gas market, including: 1) load forecast uncertainty; 2) erection and removal of other transmission lines; 3) transmission expansion costs; 4) participants' market behaviors; 5) the availability and cost of fuel; 6) energy at risk; 7) EUE cost; 8) interest and inflation rates; 9) market rules and government policies [28].

In this paper, load forecast errors, gas prices, IBDR bid prices, and system component forced outage rates (FORs) are considered as uncertainties. Monte Carlo (MC) simulations are used to generate scenarios based on the probability density functions (PDFs) of uncertainties. Furthermore, load forecast errors, gas prices and IBDR bid prices are modelled by the Gaussian distribution [29]. Forced outage rates are modelled by the Binomial distribution [30]. The mathematical formulations of these PDFs are common in the literature and they are not given in detail here. For example, a scenario can be: "for $t=1$, IBDR bid price is $\$ 70 / \mathrm{MWh}$, load level is $2000 \mathrm{MW}$, gas price is $\$ 8 / \mathrm{GJ}$, and all components are available." The convergence criterion of MC simulations is that the ratio of the standard deviation against the expected value is smaller than a pre-defined threshold.

The decision analysis is adopted in this paper to inspect various planning alternatives robustness under a series of uncertainties mentioned above. And the uncertainties are denoted by various scenarios in this paper. As for the exposed risks brought by all kinds of uncertainties, they are evaluated by means of regret. Regret is a measure of risk. For a particular future, regret is the difference between the value of an attribute for a particular plan, and the value of that attribute for the optimal plan for that future. Regret is zero for a robust plan, which indicates the optimal property of the robust plan. If a robust plan does not exist, one should choose a plan, which minimizes the maximum/average regret, or maximizes the maximum benefits, etc., depending on one's tolerance for risk [31].

\subsection{Solution method}

Our ECP model comprises of natural gas systems and power systems bounded by a variety of nonlinear constraints. Given its mixed integer nonlinear optimization characteristic, this problem is quite difficult to be solved by conventional mathematical programming techniques. A modified differential evolution (DE) algorithm with fitness sharing is used here to find the maximum benefit planning solution. The details of using enhanced DE to solve the objective function are denoted in Fig. 2. Different from conventional DE algorithm, the enhanced DE with sharing function method is capable of improving the search capability of DE while maintaining the search diversity. More details of enhanced DE with fitness sharing can be found in [32]. The program is executed on a 4 core, 64-bit DELL Desktop with Intel Core i5-2400 CPU and RAM 4 GB.

\section{Case study}

The proposed expansion co-planning model is studied on a six-bus power system, plus a seven-node gas system and a modified IEEE 118-bus system with 14-node gas system. In order to demonstrate the effectiveness of

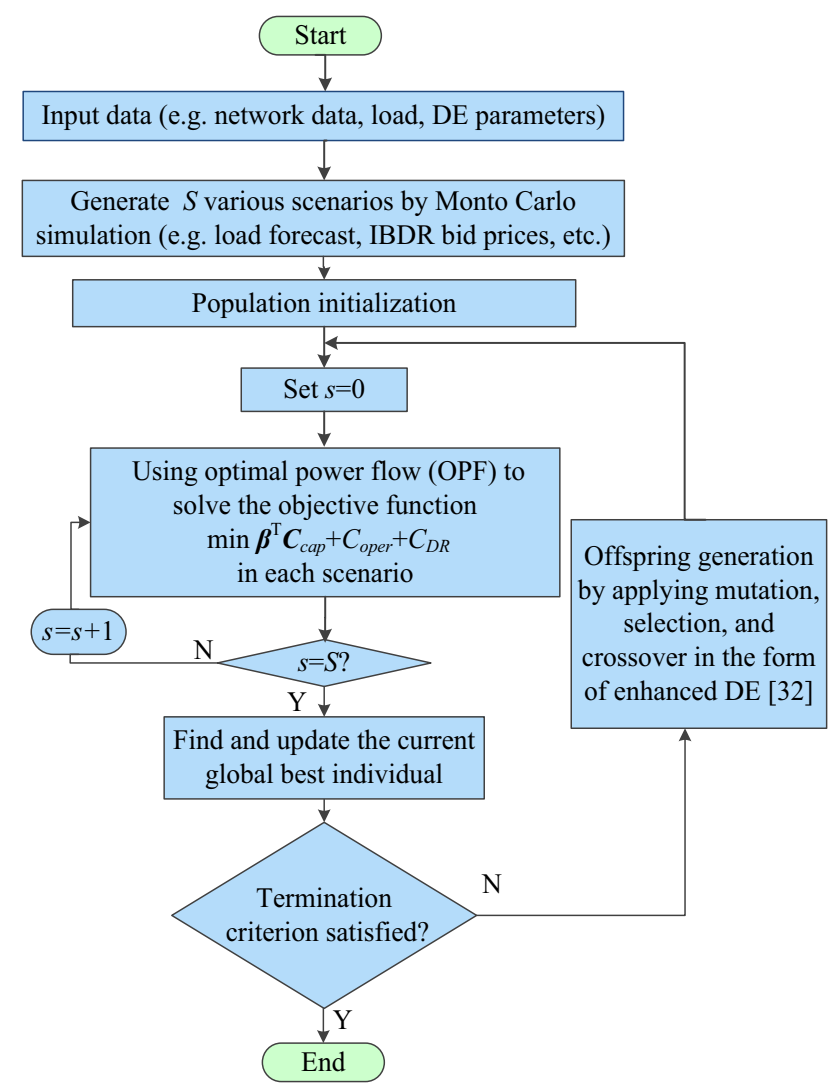

Fig. 2 Flow chart of enhanced DE algorithm for solving the coplanning model 
proposed approach, results from separated natural gas and power system planning are compared. We use various scenarios to represent the potential uncertainties in the case study. It should be noted that the annual investment costs for the four elements in the planning process (i.e. GPG units, compressor stations, gas pipelines and electricity transmission lines) are defined as $\$ 120000 / \mathrm{MW}, \$ 96000 /$ MW, $\$ 60000 / \mathrm{km}$ and $\$ 54000 / \mathrm{km}$, respectively. EUE criterion is $0.024 \%$. The transmission capacity for gas pipeline candidates is $12 \mathrm{TJ} / \mathrm{h}$ and $120 \mathrm{MW}$ for electricity transmission line candidates.

\subsection{Six-bus power system and seven-node gas system}

Figure 3 shows the one line diagram of coupled six-bus power system and seven-node gas system, which consists of three gas-fired power generation units, three electricity loads (EL), five gas loads (GL), one gas compressor (GC) and one gas storage (GS). The systems are connected by gas power plans or load centers. The gas load and electricity load variations in one day are denoted in Fig. 4. For more detailed experiment data, such as network and generation parameters, please refer to [33, 34].

The timeline scale mismatch is compensated by the variations of linepack, which makes it necessary to simulate its daily change. Note that in a gas system, the starting point of linepack should equal to the ending point of linepack. Assuming the load level increases by $15 \%$ during the planning horizon, the daily linepack changes for separated gas and power system expansion planning and integrated ECP model are shown in Fig. 5.

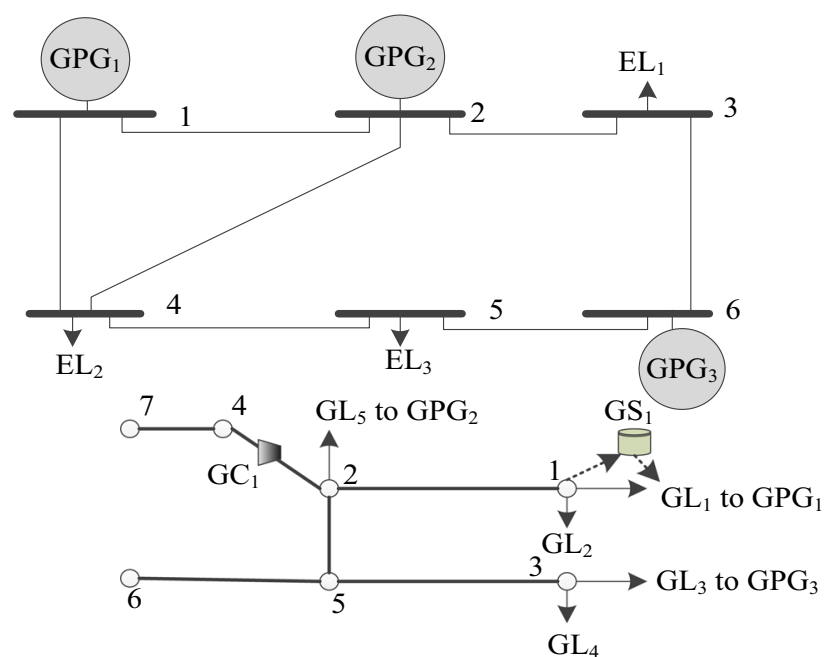

EL: electricity load; GL: gas load

GC: gas compressor; GS: gas storage

Fig. 3 One line diagram of six-bus power system and seven-node gas network

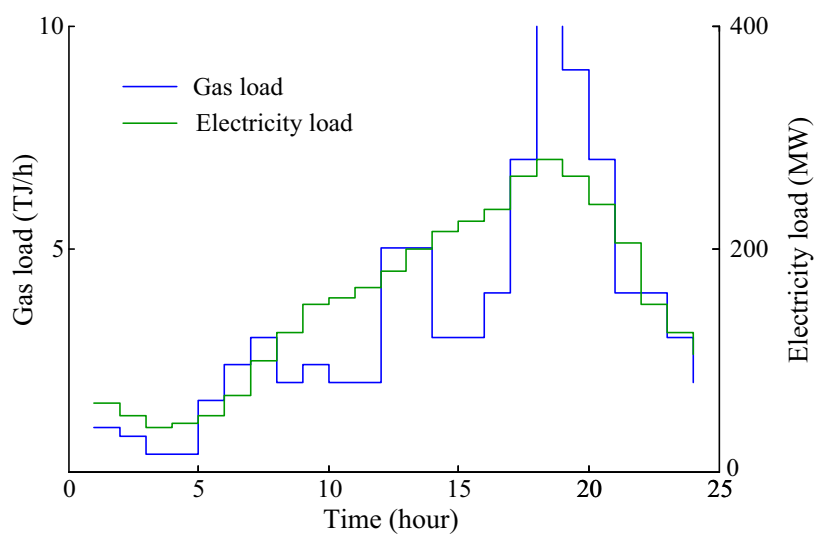

Fig. 4 Electricity load and gas load change in one day

Figure 6 illustrates the demand response and energy savings under EP and ECP conditions. It can be observed that more energy savings are derived from the perspective of ECP, which also demonstrates the economics of the proposed approach (as shown in Fig. 7).

In order to find the best augmentation plan, the following steps are needed.

In this case study, we define four scenarios to represent the potential uncertainties (i.e., S1: low gas/IBDR bid price and low load growth rate, S2: high gas/IBDR bid price and low load growth rate, S3: low gas/IBDR bid price and high load growth rate, S4: high gas/IBDR bid price and high load growth rate). At the beginning of the steps, several augmentation plans are listed. By eliminating the plans which do not meet the planning criteria, four plans are left, denoted as P1, P2, P3 and P4. The decision analysis is undertaken among various augmentation plans to find the best one. The simulation results are shown in terms of EP gas sector, EP power sector and ECP, denoted in Fig. 8.

The final decision is made by choosing the minimum value of maximum regrets. As observed from Fig. 8, the best augmentation plan is hence determined as EP gas P1,

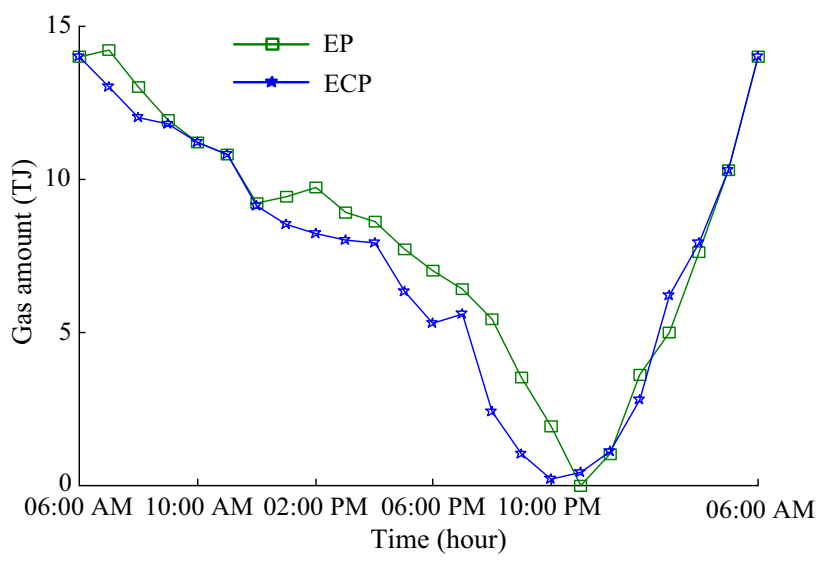

Fig. 5 Linepack variations for EP and ECP 


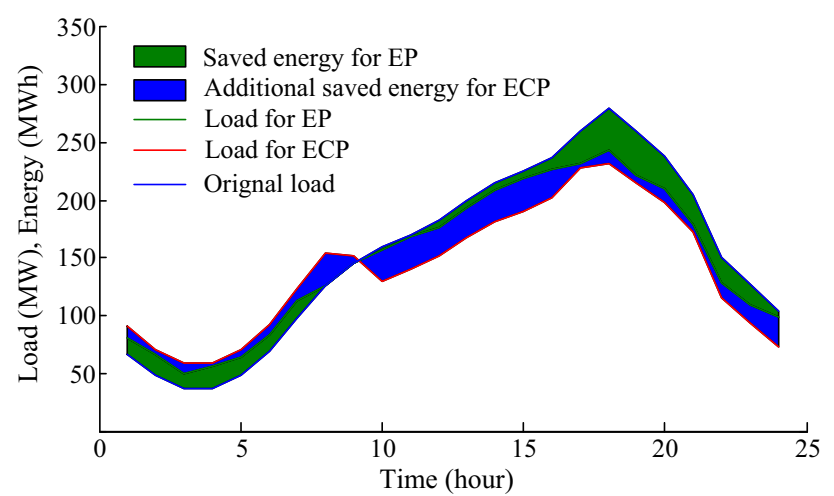

Fig. 6 Demand response and energy savings under EP and ECP conditions

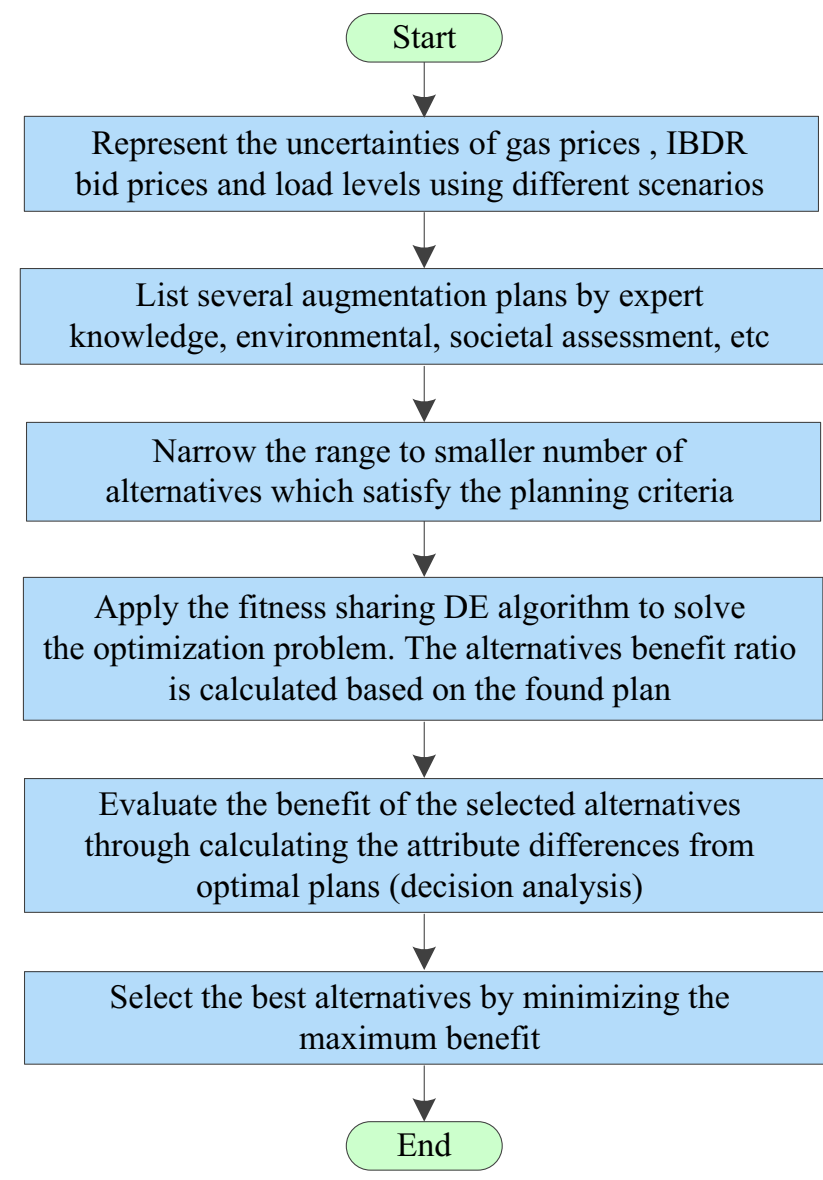

Fig. 7 Flow chart of execution steps for best augmentation plans

EP power P3 and ECP P4. Figure 9 shows the selected augmentation plans in the six-bus seven-node coupled system for EP and ECP. Compared with the traditional planning method which aims to minimize the investment and operation costs with reliability criterion satisfied, our proposed approach quantifies the system performance and expresses the benefits of boosted reliability and operation saving.

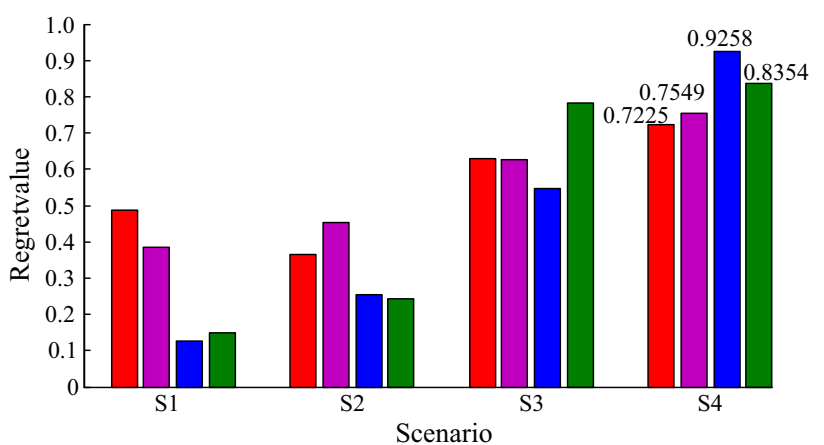

(a) EPgassector

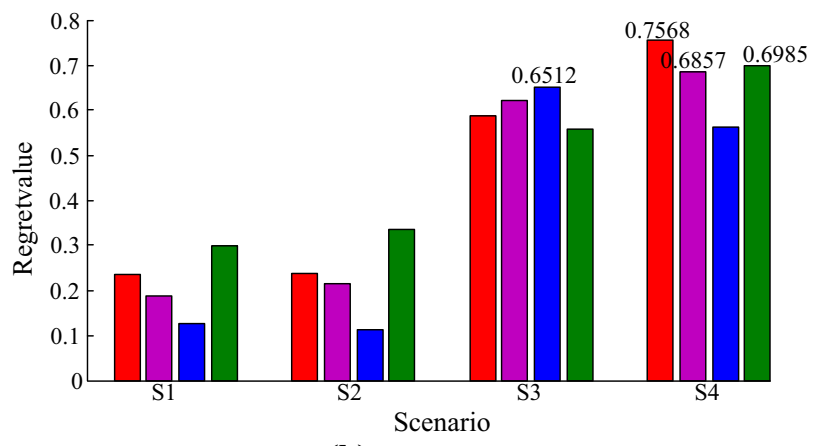

(b) EPpowersector

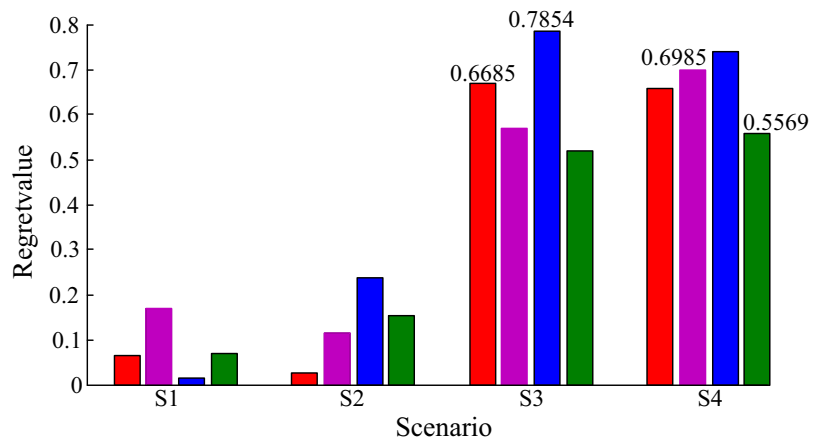

(c) ECP

$\square \mathrm{P} 1 ; \square \mathrm{P} 2 ; \square \mathrm{P}$; $\square \mathrm{P} 4$

Fig. 8 Regret values under different scenarios

The benefits of planning natural gas and power system separately through EP gas P1 and EP power P3 are quantified as follows: the investment cost is $\$ 31.87$ million; the benefit in operation is $\$ 2.608$ million; the benefit in demand response is $\$ 3.84$ million; the benefit ratio is 0.2023 .

As for the ECP approach with P4, the benefits are quantified as: the investment is $\$ 27.376$ million; the benefit in operation is $\$ 8.118$ million; the benefit in demand response is $\$ 7.298$ million; the benefit ratio is 0.5631 .

Obviously, ECP is more advantageous than EP. The investment costs are lower in ECP compared with EP, while the benefits in operation and demand response are higher in ECP. It is worth noting that the benefit ratio in 

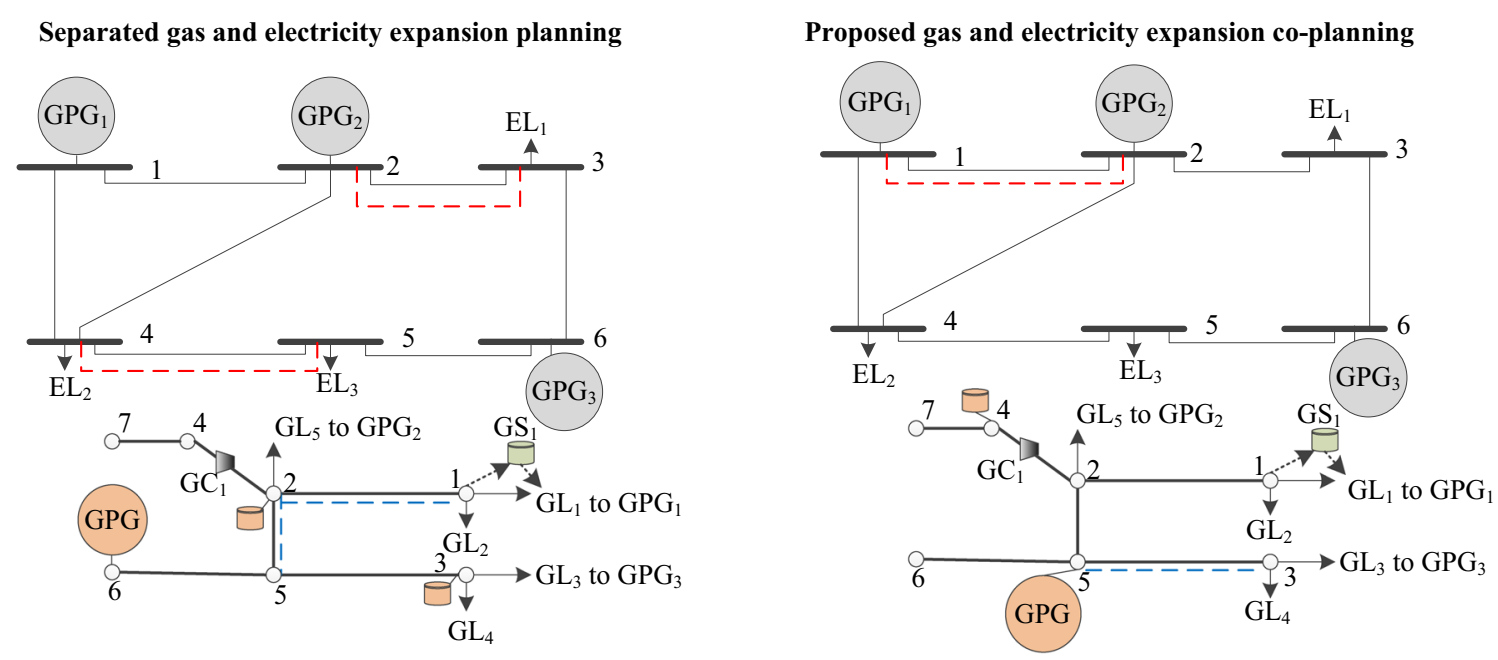

Candidate compressor; GPG Candidate GPG unit; - - - - - Candidate gas pipeline; - - - - - Candidate electricity line

Fig. 9 Chosen augmentation plans in the coupled system for EP and ECP

ECP is more than twice higher than the benefit ratio in EP. By the simulation results, we can see the ECP achieves a higher economic efficiency through the analysis of cost benefits.

\subsection{Modified IEEE 118-bus system and 14-node gas system}

The proposed approach is tested on a modified IEEE 118-bus system and 14-node gas system as well. There are 118 buses, 54 units and 186 branches in the power system, while there are two gas suppliers, two gas compressors, and 14 branches in the gas system. Other electricity and gas network parameters can be found in [33] and [34]. Assuming the forced outage rates of power lines/gas pipelines and generating units are $1 \%$ and $2 \%$ respectively. Furthermore, annual load growth is set as 5\%.

The detailed expansion plans for EP and ECP in this case study are shown in Table 1. As observed from Table 1, the total expansion cost in ECP is lower than the traditional EP approach, which demonstrates the economics of the proposed co-planning approach. Moreover, the proposed co-planning ensures the access to affordable primary gas sources and secondary electricity sources, improving the overall efficiency of energy grids. The weakness and congestion consisting in gas and electricity networks are also able to be identified and regulated strategically for the sake of meeting the overall social benefits.
Table 1 Detailed expansion plans for EP and ECP

\begin{tabular}{|c|c|c|c|c|}
\hline \multirow{2}{*}{$\begin{array}{l}\text { Expansion } \\
\text { plans }\end{array}$} & \multicolumn{2}{|l|}{ EP } & \multicolumn{2}{|l|}{ ECP } \\
\hline & Node & Capacity & Node & Capacity \\
\hline \multirow[t]{3}{*}{ GPG units } & 43 & $25 \mathrm{MW}$ & 52 & $75 \mathrm{MW}$ \\
\hline & 64 & $50 \mathrm{MW}$ & 74 & $25 \mathrm{MW}$ \\
\hline & 98 & $50 \mathrm{MW}$ & & \\
\hline \multirow[t]{3}{*}{ Gas pipelines } & $3-12$ & $42 \mathrm{~km}$ & $6-5$ & $31 \mathrm{~km}$ \\
\hline & $2-3$ & $58 \mathrm{~km}$ & $7-6$ & $52 \mathrm{~km}$ \\
\hline & $7-6$ & $86 \mathrm{~km}$ & $6-13$ & $70 \mathrm{~km}$ \\
\hline \multirow[t]{3}{*}{ Power lines } & $53-54$ & $84 \mathrm{~km}$ & $60-62$ & $54 \mathrm{~km}$ \\
\hline & $46-62$ & $102 \mathrm{~km}$ & 80-98 & $123 \mathrm{~km}$ \\
\hline & $85-93$ & 116 km & $91-92$ & $65 \mathrm{~km}$ \\
\hline Total cost & $\$ 28.2$ billion & & $\$ 27.3$ billion & \\
\hline
\end{tabular}

\section{Conclusion}

With growing global concerns for climate change, natural gas is drawing increasing attentions these years as an important type of clean fuel. Given the worldwide carbon pricing policies, increasing GPG units are built to cope with the urgent requirement for natural gas. A variety of planning issues may hence rise in regard to the reliability and security of transmission networks. Therefore, it is necessary to co-plan the GPG units with power systems in an integrated manner. An integrated ECP for natural gas 
and power system is proposed in this paper, which is formulated as a mixed integer nonlinear problem. The objective of the proposed approach is to find the best augmentation plan with the minimum cost. In addition, the variations of linepack are simulated to compensate the timeline mismatch of natural gas and electricity.

The proposed ECP model is tested on a coupled six-bus power system with seven-node gas network, and a modified IEEE 118-bus system with 14-node gas system. The simulation results demonstrate that our model is advantageous as threefold: 1) It can meet the energy request in the long way by identifying the energy infrastructure shortcomings; 2) It can support system planning decisions and understand the interactive activities between gas networks and power systems; 3) It can be more cost effective to contribute to the whole society.

Acknowledgements This work was supported in part by funding from the Faculty of Engineering \& Information Technologies, The University of Sydney, under the Mid-career Researcher Development Scheme, in part by the ARC Discovery Grant (No. DP170103427), and in part by the 2015 Science and Technology Project of China Southern Power Grid (No. WYKJ00000027).

Open Access This article is distributed under the terms of the Creative Commons Attribution 4.0 International License (http:// creativecommons.org/licenses/by/4.0/), which permits unrestricted use, distribution, and reproduction in any medium, provided you give appropriate credit to the original author(s) and the source, provide a link to the Creative Commons license, and indicate if changes were made.

\section{References}

[1] Zhou X, James G, Liebman A et al (2010) Partial carbon permits allocation of potential emission trading scheme in Australian electricity market. IEEE Trans Power Syst 25(1):543-553

[2] Liu G, Zhao J, Wen F et al (2010) Option-game-based method for generation investment analysis considering uncertain carbon reduction policy in the electricity market. IET Gen Transm Distrib 4(8):917-927

[3] Taggart S, James G, Dong $\mathrm{Z}$ et al (2012) The future of renewables linked by a Transmissional Asian Grid. Proc IEEE 100(2):348-359

[4] Urbina M, Li Z (2007) A combined model for analyzing the interdependency of electrical and gas systems. In: Proceedings of the IEEE 39th North American power symposium, pp 468-472

[5] Tao L, Eremia M, Shahidehpour M (2008) Interdependency of natural gas network and power system security. IEEE Trans Power Syst 23(4):1817-1824

[6] An S, Li Q, Gedra T (2003) Natural gas and electricity optimal power flow. In: Proceedings of the IEEE PES transmission and distribution conference and exposition, Dallas, TX, USA, 7-12 September 2003, pp 138-143

[7] Martinez A, Fuerte C (2012) A unified gas and power flow analysis in natural gas and electricity coupled networks. IEEE Trans Power Syst 27(4):2156-2166
[8] Chaudry M, Jenkins N, Strbac G (2008) Multi-time period combined gas and electricity network optimisation. Electr Power Syst Res 78(7):1265-1279

[9] Mads A, Ian H (2011) Optimization framework for the analysis of large-scale networks of energy hubs. In: Proceedings of the 17 th power systems computation conference, Stockholm, Sweden, 22-26 August 2011

[10] Wu L, Shahidehpur M (2011) Optimal coordination of stochastic hydro and natural gas supplies in midterm operation of power systems. IET Gen Trans Distrib 24(3):1523-1536

[11] Shukla P, Dhar S, Victor D et al (2009) Assessment of demand for natural gas from the electricity sector in India. Energy Policy 37(9):3520-3534

[12] Kabirian A, Hemmati M (2007) A strategic planning model for natural gas transmission networks. Energy Policy 35(11):5656-5670

[13] Roh J, Shahidehpour M, Yong F (2007) Market-based coordination of transmission and generation capacity planning. IEEE Trans Power Syst 22(4):1406-1419

[14] Sharan I, Balasubramanian R (2012) Integrated generation and transmission expansion planning including power and fuel transportation constraints. Energy Policy 43:275-284

[15] Tekiner H, Coit D, Felder F (2010) Multi-period multi-objective electricity generation expansion planning problem with MonteCarlo simulation. Electr Power Syst Res 80(12):1394-1405

[16] Unsihuay C, Marangon J, Souza A (2010) A model to long-term, multistage, and integrated expansion planning of electricity and natural gas sytems. IEEE Trans Power Syst 25(2):1154-1168

[17] Qiu J, Dong Z, Zhao J et al (2014) Expansion co-planning with uncertainties in a coupled energy market. In: Proceedings of the IEEE PES general meeting, Washington, USA, 27-31 July 2014, pp 1-5

[18] Qiu J, Dong Z, Zhao J et al (2015) Expansion co-planning for shale gas integration in a combined energy market. J Mod Power Syst Clean Energy 3(3):302-311. doi:10.1007/s40565-0150107-1

[19] Wang D, Meng K, Gao X et al (2017) Optimal air conditioning load control in distribution network with intermittent renewables. J Mod Power Syst Clean Energy 5(1):55-65. doi:10.1007/ s40565-016-0254-Z

[20] Qiu J, Dong Z, Meng K et al (2016) Multi-objective transmission expansion planning in a smart grid using a decompositionbased evolutionary algorithm. IET Gen Transm Distrib 10(16):4024-4031

[21] Chen S, Liu C (2017) From demand response to transactive energy: state of the art. J Mod Power Syst Clean Energy 5(1):10-19. doi:10.1007/s40565-016-0256-x

[22] Australian Energy Market Operator (AEMO) (Online). http:// www.aemo.com.au/. Accessed 13 Dec 2016

[23] Shahidehpour M, Fu Y, Wiedman T (2005) Impact of natural gas infrastructure on electric power systems. Proc IEEE 93(5):1042-1056

[24] Munoz C, Sauma E et al (2012) Impact of high wind power penetration on transmission network expansion planning. IET Gen Trans Dist 6(12):1281-1291

[25] Nguyen D, Negnevitsky M, Groot M (2013) Market-based demand response scheduling in a deregulated environment. IEEE Trans Smart Grid 4(4):1948-1956

[26] Mashhour E, Moghaddas-Tafreshi S (2011) Bidding strategy of virtual power plant for participating in energy and spinning reserve markets-part I: problem formulation. IEEE Trans Power Syst 26(2):949-956

[27] Zeng Q, Fang J, Li J et al (2016) Steady-state analysis of the integrated natural gas and electric power system with bi-directional energy conversion. Appl Energy 185:1483-1492 
[28] Zhao J, Dong Z, Lindsay P et al (2009) Flexible transmission expansion planing with uncertainties in an electricity market. IEEE Trans Power Syst 24(1):479-488

[29] Li C, Dong Z, Chen G et al (2015) Flexible transmission expansion planning associated with large-scale wind farms integration considering demand response. IET Gen Trans Distrib 9(15):2276-2283

[30] Billinton R, Allan R (1996) Reliability evaluation of power systems, 2nd edn. Plenum Press, New York

[31] Torre T, Feltes J, Roman T et al (1999) Deregulation, privatization, and competition: transmission planning under uncertainty. IEEE Trans Power Syst 14(2):460-465

[32] Yang G, Dong Z, Wong K (2008) A modified differential evolution algorithm with fitness sharing for power system planning. IEEE Trans Power Syst 24(1):479-488

[33] Liu C, Shahidehpour M, Fu Y et al (2009) Security-constrained unit commitment with natural gas transmission constraints. IEEE Trans Power Syst 24(3):1523-1536

[34] Khodaei A, Shahidehpour M, Kamalinia S (2010) Transmission switching in expansion planning. IEEE Trans Power Syst 25(3):1722-1733

Dongxiao WANG received the bachelor degree from North China Electric Power University, Beijing, China, in 2014. He is currently pursuing the Ph.D. degree at the Centre for Intelligent Electricity Networks, the University of Newcastle, Australia. His research interest includes demand side management, the utilization of thermostatically controlled loads, energy storage systems and renewable energy.

Jing QIU received his Ph.D. degree from the University of Newcastle, Australia in 2014. He is now a Research Associate at the Centre for Intelligent Electricity Networks, the University of Newcastle, Australia. His research interests include electricity modelling, power system planning, renewable energy, carbon emission reduction, and risk management.

Ke MENG received the Ph.D. degree from the University of Queensland, Brisbane, QLD, Australia, in 2009. He is currently with the School of Electrical and Information Engineering, The University of Sydney, Sydney, NSW, Australia. He is also a Visiting Professor at the Changsha University of Science and Technology, Changsha, China. His research interests include power system stability analysis, power system planning, renewable energy, and energy storage.

Xiaodan GAO received the bachelor degree from North China Electric Power University, Beijing, China, in 2013. She is currently pursuing the Ph.D. degree at the Centre for Intelligent Electricity Networks, the University of Newcastle, Australia. Her research interest includes wind power integration, wind turbine control, and distribution system control.

Zhaoyang DONG obtained his Ph.D. degree from the University of Sydney, Australia in 1999. He is now Professor and Head of School of Electrical and Information Engineering, University of Sydney, Australia. He is also a Visiting Professor at the Changsha University of Science and Technology, Changsha, China. He is immediate Ausgrid Chair Professor and Director of the Centre for Intelligent Electricity Networks (CIEN), the University of Newcastle, Australia. He previously held academic and industrial positions with the Hong Kong Polytechnic University, the University of Queensland, Australia and Transend Networks, Australia. His research interest includes smart grid, power system planning, power system security, load modeling, renewable energy systems, electricity market, and computational intelligence and its application in power engineering. He is an editor of IEEE Transactions on Smart Grid, IEEE Transactions on Sustainable Energy, IET Renewable Power Generation, and IEEE Power Engineering Letters. 